\title{
Unpredictable pain timings lead to greater pain when people are highly intolerant of uncertainty
}

Clémence Bélanger, M.PT. ${ }^{\text {, }}$, Bernard Blais Morin, M.PT. . , Andréanne Brousseau, M.PT.. , Nicolas Gagné, M.PT. ${ }^{\mathrm{a}}$, Anne Tremblay, M.PT. ${ }^{\mathrm{a}}$, Kathya Daigle, M.Sc. ${ }^{\mathrm{a}}$, Philippe Goffaux ${ }^{\mathrm{b}}$, Ph.D. ${ }^{\mathrm{a}}$, Guillaume Léonard, PT., PhD.* *a,

${ }^{a}$ School of Rehabilitation, Faculty of Medicine and Health Sciences, Université de Sherbrooke, Sherbrooke, QC, Canada, J1H 5N4; ${ }^{b}$ Department of Surgery, Faculty of Medicine and Health Sciences, Université de Sherbrooke, Sherbrooke, QC, Canada, J1H 5N4; ${ }^{\mathrm{c}}$ Research Center on Aging, CIUSSS de 1'Estrie-CHUS, Sherbroooke, QC, Canada, J1H 4C4

*Corresponding author: Guillaume Léonard, PT., Ph.D.

Research Center on Aging

CIUSSS de l'Estrie-CHUS

1036, rue Belvédère Sud

Sherbrooke (Québec) J1H 4C4 


\begin{abstract}
Background and purpose: Many psychological factors are known to influence pain perception. Among them, intolerance of uncertainty (IU) may play a key modulating role in situations where uncertainty prevails, especially uncertainty regarding the timing of painful events. The objective of this study was to explore the impact of individual differences in IU on pain perception during predictable and unpredictable stimulation timings. We hypothesized that people with high IU, as opposed to those with low IU, would perceive more pain when the timing of painful stimulations cannot be predicted, as compared to when they can.

Methods: Twenty (20) healthy adults, aged between 18 and 35 years old, were recruited. Painful sensations were provoked using transcutaneous electrical stimulations of the right sural nerve. By measuring IU (Intolerance of Uncertainty Scale) and subjective pain (verbal numerical rating scale), it was possible to test the relationship between IU and pain perception, by simulating predictable and unpredictable painful experiences. This was done through cued shock interval (CSI) blocks, with either variable timing or fixed timings (long or short time frame). Selfadministered questionnaires were also used to measure pain hypervigilance, pain catastrophising, state anxiety, and trait anxiety.

Results: Pearson correlations confirmed the presence of an association $(r=0.63)$ between IU and the change in pain intensity provoked by unpredictable stimulation timings. Importantly, this association was significant only for stimulations provided at long CSIs, indicating that higher IU scores predicted higher pain intensity scores when stimulation timings became unpredictable, and when the cued delay was long. No association was found between pain scores and other psychological variables.

Conclusions: Our results show that IU moderately correlates to the change in pain intensity provoked by unpredictable stimulation timings. High IU scores were associated with a worsening of the subjective pain experience, especially during long delays in an unpredictable situation. These observations suggest that IU could be considered as a psychological variable that is able to influence pain perception in certain situations.
\end{abstract}

Implications: Assessing and addressing IU could be an added value in pain-related therapy, and especially in chronic pain.

\title{
Key words
}

Pain; intolerance of uncertainty; anxiety; hypervigilance; experimental pain 


\section{Introduction}

Many factors, including psychological ones, influence pain perception [1-4]. Anxiety, hypervigilance to pain, and pain catastrophizing have been proven to influence pain perception [5-9]. Another psychological factor that may play a role in pain perception is intolerance of uncertainty (IU). This psychological construct can be defined as the tendency to respond to uncertain situations or events with negative emotional, cognitive, and behavioural reactions [10]. The absence of literature linking pain perception and intolerance of uncertainty shows that IU is a putative pain-related psychological factor which remains poorly studied. Previous studies have demonstrated that subjects who are intolerant of uncertainty are both more anxious [11] and more attentive to potentially dangerous situations [12], two predispositions which are known to heighten the subjective experience of pain [5,13]. IU, therefore, may be an important factor in explaining why humans differ so much in their response to pain. Specifically, IU may play a modulating role in situations where uncertainty prevails, especially when uncertainty regarding the timing of painful events abounds $[14,15]$. To indicate the time of onset of the stimulation, and its predictability or non-predictability, many types of cueing designs can be used, such as sound cueing and visual cueing $[11,14,16]$. Both variations (predictability and length of the delay before the shock) have shown their influence on pain perception $[14,15,17]$. Indeed, recent studies confirm that when the timing of pain cannot be fully predicted, some react quite poorly and report increased pain $[14,15]$. To this day, there is no consensus on the best way to create an unpredictable environment in experimental settings.

Understanding why some of us react so poorly to unpredictable pain, while others do not, may require that we pay closer attention to innate predispositions regarding IU. The objective of this study was to explore the impact of individual differences in IU on pain perception, during predictable and unpredictable stimulation timings. We hypothesized that people with high IU, as opposed to those with low IU, would perceive more pain when the timing of painful stimulations cannot be predicted, as compared to when they can.

\section{Materials and Methods}

\section{$\underline{2.1 \text { Participants }}$}


Twenty (20) healthy adults between the ages of 18 and 35, including 10 men (mean age $22.5 \pm$ 2.4 years) took part in this study. All participants provided written, informed consent, and the research protocol was approved by the ethics committee of the Centre Hospitalier Universitaire de Sherbrooke (CHUS).

\subsection{Subjective pain intensity}

Pain intensity was assessed using a verbal numerical rating scale (NRS). The scale ranged from $0-100$, where 0 was defined as "no pain" and 100 was defined as "intolerable pain intensity". Numerical rating scales have excellent psychometric properties, and are very sensitive to minimally experienced changes in subjective pain [18].

\subsection{Sural nerve stimulation}

Painful sensations were provoked using transcutaneous electrical stimulations of the right sural nerve. The sural nerve was stimulated over its retromalleolar path. Stimulations of the sural nerve consisted in 10 electrical impulses with a wavelength of $1 \mathrm{~ms}$ and a frequency of $240 \mathrm{~Hz}$. Prior to testing, a pre-experimental session was carried out to help participants become familiar with all electrical stimulations, to determine their pain threshold level, and to identify the stimulation intensity required to provoke a sensation of 30/100 pain (i.e., suprathreshold pain sensitivity). During the testing phase of the experiment, electrical stimulations of the sural nerve were always cued ahead of time, using a visual cue (red light) which signaled the presence of an upcoming shock. Subjects were seated $100 \mathrm{~cm}$ from the visual cue (home-made stimulus box containing a red light-emitting diode placed behind a translucent circular screen, 2 degrees in diameter). The visual cue was turned off one second after stimulus onset, and remained turned off until the next trial, which occurred 6 sec later. Participants were asked to provide their pain ratings during this $6 \mathrm{sec}$ cue-off interval. A depiction of the cueing design is presented in Figure 1. Shocks were administered in 3 separate testing blocks: 2 fixed blocks and 1 variable block. Each fixed block contained 8 sural nerve stimulations. In fixed blocks, shocks were always presented after a fixed delay following cue onset. The cue shock interval (CSI) was always the same length in a given fixed block, either 6 or $15 \mathrm{sec}$, depending on the block. In the variable block, shocks were presented after a variable delay following cue onset. The CSI in the variable block varied between 6, 9, 12 and 15 seconds. In this block, 4 sural nerve shocks per CSI were 
presented, for a total of 16 stimulations. CSIs within the variable block were always presented using the same, pre-established, random sequence. Participants were told that shocks would always be cued ahead of time, and that the CSI would remain fixed within the fixed blocks, and variable within the variable block. Regardless of block type, CSI length was never directly specified. Participants were also never told that the stimulation intensity would be kept constant throughout testing (at a stimulation intensity value required to provoke 30/100 pain). To ensure homogeneity between the group receiving variable shocks first, and the group receiving the fixed block first, the participants in these groups were matched for sex and IU score.

\section{(Insert Figure 1)}

It is important to point out that psychological variables other than IU, such as anxiety, hypervigilance to pain, and pain catastrophizing, may further contribute to the pain enhancing effects of unpredictable timing. See for instance Ruscheweyh et al. who demonstrate the enhancing effect of pain catastrophizing on pain perception [9]. To properly identify the unique contribution of each of these variables to the putative pain enhancing effects of unpredictable timing, and because these variables may explain pain enhancement as well as (or better than) IU, we included them as predictor variables in the present study.

\subsection{Questionnaires}

\subsubsection{Intolerance of uncertainty}

IU was measured using the Intolerance of Uncertainty Scale (IUS) [19]. The IUS was developed to assess emotional, cognitive, and behavioral reactions to ambiguous situations, implications of being uncertain, and attempts to control the future [20]. It shows excellent internal consistency and good test-retest reliability [19,20]. The IUS is a 27-item questionnaire scored on a 5-point Likert scale. The IUS score varies from 27 to 135 points, where high scores represent higher levels of uncertainty.

\subsubsection{Anxiety}


The State-Trait Anxiety Inventory (STAI) was used to measure anxiety. The STAI consists in 2 20-item questionnaires scored using a 4-point Likert scale. The STAI measures a standard index of situational $($ state $=$ STAI-S) and dispositional (trait $=$ STAIT-T) anxiety. The STAI has excellent psychometric properties [5,21] and is frequently used in pain research [22-24]. High scores on the STAI (state or trait) indicate elevated levels of anxiety.

\subsubsection{Pain catastrophizing}

The Pain Catastrophizing Scale (PCS) was used to measure catastrophizing thoughts related to pain. The PCS is a 13-item questionnaire using a 5-point Likert scale. Each question is part of 1 of the 3 subsections related to a construct evaluated by the PCS: rumination, magnification, and helplessness. The total score on the PCS is calculated by summing all the items (minimum score $=13$, maximum score $=52$ ). An elevated score indicates elevated catastrophic thinking. The PCS has excellent psychometric properties $[22,25]$ and is frequently used in pain research.

\subsubsection{Pain Hypervigilance and Awareness Questionnaire (PVAQ)}

The Pain Hypervigilance and Awareness Questionnaire (PVAQ) was used to measure attention to pain. This scale consists in 16 items rated on a 6-point scale regarding awareness, vigilance, observation, and preoccupation with pain. PVAQ is a valid and reliable questionnaire [26]. High scores on the PVAQ indicate a greater attention to pain [27].

\subsection{Experimental Protocol}

Participants were first asked to read and sign the consent form, and then completed autoadministered questionnaires. Participants were then seated comfortably in a reclining chair facing the visual cue. The testing room was slightly dark, and the chair was positioned in a way so that the participant experienced minimum distractions. The skin behind the left external malleoli was exfoliated and disinfected with alcohol to assure proper contact between the stimulator and the skin. Once the electrode was installed, the pre-testing phase began. During this phase, shocks of growing intensity were delivered to determine the stimulation intensity that would be used during the testing phase (targeted initial pain intensity $=30 / 100$ ). The participants were exposed to painful stimulation of a maximum of 60/100 during this phase, which allowed them to have a better knowledge of the pain scale. The actual testing phase followed. This phase, which lasted 
no more than 20 minutes, included the 3 testing blocks, as explained above. To reduce expectations, participants were told that the intensity of the electrical shocks could vary. A 2minute break was allowed between each block, to avoid pain sensitization. Once the pain and anxiety scores were collected for each shock of each 3 blocks, the subject was thanked for his participation, and received a \$20 compensation to cover parking and travelling fees.

\subsection{Statistical Analyses}

\subsubsection{Effect of unpredictable stimulation timing on subjective pain intensity}

Two (2) separate mixed design ANOVAs were conducted to explore the effects of unpredictable stimulation timing on subjective pain intensity. The first ANOVA was conducted for shocks

provided with short delays (i.e., $6 \mathrm{sec}$ ) and the second ANOVA was conducted for shocks with long delays (i.e., $15 \mathrm{sec}$ ). In both ANOVAs, the independent variables were Predictability (Fixed block vs Variable block), Block order (Fixed block first vs Variable block first) and Fixed block order (Fixed block $6 \mathrm{sec}$ first vs Fixed block $15 \mathrm{sec}$ first). The dependent variable was mean pain intensity (collapsed across stimulation trials).

\subsubsection{Effect of stimulation timing delay (within the variable timing block) on subjective pain} intensity

A mixed design ANOVA was conducted to explore the effects of stimulation timing delay (in the variable block) on subjective pain intensity. Independent variables included Timing (6 vs 9 vs 12 vs $15 \mathrm{sec}$ ) and Block order (Fixed block first vs Variable block first). Fixed block order (Fixed block $6 \mathrm{sec}$ first $v s$ Fixed block $15 \mathrm{sec}$ first) was not included as an independent variable, since the data from fixed blocks were not considered in this analysis. The dependent variable was mean pain intensity (collapsed across stimulation trials). For all ANOVAs, Greenhouse-Geisser corrections for non-sphericity were applied where appropriate, and Bonferroni corrections were applied to all post-hoc analyses requiring multiple comparisons. Given our relatively small sample size, a measure of effect size $\left(\eta^{2}\right)$ was also systematically reported.

\subsubsection{Correlation analyses}


To explore the relationship between the key predictor variables (i.e., IU, anxiety, hypervigilance to pain, and pain catastrophizing) and the psychophysical pain indices (i.e., pain threshold, suprathreshold pain sensitivity, and change in pain intensity provoked by unpredictable stimulation timings), a series of Pearson product-moment correlations were done. It is important to note that the change in pain intensity provoked by unpredictable stimulation timings was calculated by subtracting the NRS pain score experienced in the predictable timing block from the pain experienced in the unpredictable timing block. This delta score was calculated twice, namely once for shocks provided at $6 \mathrm{sec}$ delays, and once for shocks provided at $15 \mathrm{sec}$ delays. Thus, positive delta scores reflect the experience of greater pain during unpredictable, as opposed to predictable, timing blocks. Given the large number of correlations that were run, and given the over-conservative nature of Bonferroni corrections under such conditions, we favoured a less stringent approach, namely that of setting the conceptual unit of error (alpha level) at 0.005. In our opinion, this approach reduces the risk of committing both a type I and/or a type II error.

\subsubsection{Non-parametric tests}

Because of the relatively small number of subjects included in the study, and despite histograms suggesting the presence of normally distributed data, nonparametric tests were added. Results confirmed no difference between parametric and nonparametric approaches; therefore, only parametric statistics are reported.

\section{Results}

\subsection{Effect of unpredictable stimulation timing on subjective pain intensity}

For stimulations provided at short $(6 \mathrm{sec})$ CSIs, results showed that subjective pain intensity did not differ between predictable and unpredictable stimulation timing blocks (i.e., no main effect of Predictability; $\left.\mathrm{F}_{1}, 16=0.65, \mathrm{p}=0.43 ; \eta^{2}=0.04\right)$. Moreover, there was no interaction of Predictability with Block order $\left(\mathrm{F}_{1,16}=0.19, \mathrm{p}=0.67 ; \eta^{2}=0.01\right)$, Fixed block order $\left(\mathrm{F}_{1,16}=0.34\right.$, $\left.\mathrm{p}=0.57 ; \eta^{2}=0.02\right)$ or both Block order and Fixed block order $\left(\mathrm{F}_{1,16}=1.60, \mathrm{p}=0.22 ; \eta^{2}=0.09\right)$. 
We also failed to find a main effect of Predictability for shocks provided at long (15 sec.) CSIs $\left(\mathrm{F}_{1,16}=1.61, \mathrm{p}=0.22 ; \eta^{2}=0.09\right)$. Likewise, there was no interaction of Predictability with Block order $\left(\mathrm{F}_{1,16}=0.17, \mathrm{p}=0.69 ; \eta^{2}=0.01\right)$, Fixed block order $\left(\mathrm{F}_{1,16}=4.25, \mathrm{p}=0.06 ; \eta^{2}=0.21\right)$ or both Block order and Fixed block order $\left(\mathrm{F}_{1,16}=0.50, \mathrm{p}=0.49 ; \eta^{2}=0.03\right)$ for stimulations provided at long CSIs. All subjective pain intensity values are presented in Table 1.

\section{(Insert Table 1)}

3.2 Effect of stimulation timing delay (within the variable timing block) on subjective pain intensity

Results showed that varying CSI length $(6,9,12$, or $15 \mathrm{sec})$ within variable timing blocks had no effect on subjective pain ratings (i.e., no main effect of Timing; $\mathrm{F}_{3,54}=1.85, \mathrm{p}=0.18 ; \eta^{2}=0.09$ ). Timing also did not interact with Block order $\left(\mathrm{F}_{3,54}=2.86, \mathrm{p}=0.08 ; \eta^{2}=0.14\right)$ (see Table 2).

\section{(Insert Table 2)}

\subsection{Correlation analyses}

The only significant association found between our predictor variables and our psychophysical pain indices was between IU and the change in pain intensity provoked by unpredictable stimulation timings $(\mathrm{r}=0.63, \mathrm{p}=0.003$; see entire correlation matrix in Table 3$)$. This association was significant only for stimulations provided at long CSIs, suggesting that higher IU scores predicted higher pain intensity scores when stimulation timings became unpredictable and when the cued delay was long. The relationship between IU and the change in pain intensity is depicted in Figure 2. As it can be seen from this figure, the significant and positive association noted between these two variables can possibly be attributable to the values of a few participants who showed high IU and high pain variation scores. To exclude this possibility, the association between IU and the change in pain intensity was further explored using Pearson's rank correlation coefficient. Results from this non-parametric test still revealed the presence of a significant association between IU and the change in pain intensity $(r=0.63 ; p<0.003)$. 


\section{(Insert Table 3 and Figure 2 here)}

\section{Discussion}

In the present study, nociceptive stimuli whose onset timings could not be predicted were not experienced as more painful than nociceptive stimuli whose onset timings could be fully predicted. Despite this null effect, a substantial amount of inter-individual variability in the effect of unpredictable stimulation timing on subjective pain intensity was observed, a variability which could be related to IU. More specifically, participants who were most intolerant of uncertainty were also those who experienced the greatest increase in pain when stimulation timings changed from being predictable to unpredictable. This was most obvious when shocks were provided at long cue-shock delays, as opposed to short cue-shock delays. This is consistent with the results of a prior study published by Story and colleagues which showed that, for some individuals, the presence of a long cue-stimulus delay made the pain stimulation more aversive [28]. Story and colleagues labelled those individuals "dreaders". In fact, the authors also discovered that dreaders would rather feel strong pain right away, than feel much milder pain at some later (delayed) time. Dreaders, therefore, prefer to hasten the occurrence of pain to alleviate the trepidation brought on by delaying painful experiences. This is coherent with our findings, which suggest that highly IU participants have greater pain enhancement after long delays than after short delays. These observations may be explained by the fact that long delays enhance the build-up of anxiety and thus, tend to enhance the attention paid to upcoming noxious stimuli $[11,14,29]$. Future studies are needed to better understand the differences and similarities between the dread effect and IU.

Although IU clearly shared variance with the other psychological variables, no other psychological variable was related to the increase in pain when stimulation timings became unpredictable. The fact that no other psychological variables (vigilance to pain, pain catastrophizing, trait anxiety, state anxiety) predicted significant pain enhancement, suggests something quite unique to IU. The variability observed in pain enhancement scores is not only due to the predictability of the stimulation, but is mainly explained by the individual differences in IU scores. However, as shown in Table 3, the Pearson correlation coefficient between trait anxiety and the increase in pain felt at short CSIs reached 0.60; however, the relationship 
between these 2 variables was not statistically significant. More studies with larger samples will be necessary to clarify this relationship between trait anxiety and pain enhancement.

To our knowledge, this is the first study to show that IU is related to an increase in pain when this pain is unpredictable. A major strength of our study is that we properly controlled for sequence effects, via randomization for block order and fixed block order. This way, we have ensured that people who felt more pain in the variable block did so because of the unpredictability of the stimuli rather than the simple fact that they could feel more pain at the beginning of the experimentation and then habituate to the nociceptive stimuli, or at the opposite, feel less pain first and then sensitize to the painful nociceptive. Finally, to ensure that the group of participants receiving the variable block first would be similar to the group receiving the fixed block first, we properly matched the individuals in these groups for sex and IU scores.

One limitation of this study is that we did not measure pain-related anxiety from trial-to-trial (although we did measure situational anxiety and found that it was not related to the amplification of pain caused by unpredictable stimulation timings). We cannot know if anxiety builds up during long CSIs, nor can we know if instantaneous fluctuations in anxiety shape the cost of unpredictability and/or mediate the link between IU and the amplification of pain caused by unpredictable stimulation timings. Thus, it would be interesting to repeat the current study while also measuring trial-to-trial fluctuations in pain-related anxiety. This would allow us to test the hypothesis that anxiety builds up during long CSI, and explain why the association between IU and pain amplification was present for long, but not for short CSIs. Future studies will also have to determine the delay length most suited to produce pain enhancement effects. Current studies involving delayed pain stimuli typically use delays which vary anywhere between 3 to 27 seconds [12,14,30,31] but there is currently no consensus on which length of delay is most appropriate.

Moreover, another limitation of our study is that it presents a small sample size $(\mathrm{n}=20)$ and IU scores lack variability. A future study should ensure the sampling of a larger number of participants, with a wider range of IU scores. This will surely provide a more reliable estimate of the association between IU and pain amplification. Actually, the association is sustained by a few 
participants who are highly intolerant to uncertainty. A greater number of IU participants would allow confirmation that the observed association is robust, and not only driven by a few potential outliers, and that a type I error did not occur. Furthermore, the effect of other psychological variables on pain perception could be missed because of low statistical power due to small sample size, leading to a type II error. Moreover, our sample was relatively homogenous (all healthy people between 18-35 years old), thus we cannot generalize the results to the rest of the population. It would be interesting to explore additional age cohorts, and perhaps participants who suffer from either anxiety disorders or chronic pain. Future projects could analyze the distribution of IU scores in people suffering from chronic pain, and verify if the phenomenon of pain enhancement occurs under the same conditions (high IU scores and long delays) for patients with chronic pain or under different conditions than those experimented with healthy participants.

\section{Conclusion}

This study reveals that IU moderately correlates to the change in pain intensity provoked by unpredictable stimulation timings. This finding suggests that high IU scores predicted a worsening of the subjective pain experience, especially during long delays, in an unpredictable situation. Future studies with larger samples and more variability in IU scores are needed to confirm these results, and to verify if this phenomenon, found in long delays, is due to the buildup of anxiety.

\section{Implications}

In trying to understand the role of IU on pain perception, we lay the groundwork for research aimed at better understanding chronic pain. Chronic pain is a complex phenomenon that is significantly influenced by instances where the timing of painful paroxysms is fully unpredictable. If the relationship between IU and pain perception exists, IU could be recognized as a target of investigation and intervention in people suffering from chronic pain.

\section{Financial Support}

P. Goffaux and G. Léonard are supported by the Fonds de recherche Santé (FRQS, Québec, Canada) and by the Natural Sciences and Engineering Research Council (NSERC, Canada). 
The authors have no conflicts of interest to disclose.

\section{Ethical Issues}

All participants provided written, informed consent, and the research protocol was approved by the ethics committee of the Centre Hospitalier Universitaire de Sherbrooke (CHUS). 


\section{References}

[1] Simons LE, Elman I, Borsook D. Psychological processing in chronic pain: a neural systems approach. Neurosci Biobehav Rev. 2014;39:61-78.

[2] Knotek P, Knotkova H. Psychological processes in chronic pain: A structural model. Journal of Pain Management. 2008;1:295-305.

[3] Linton S. Understanding pain for better clinical practice: a psychological perspective. Elsevier Health Sciences.; 2005.

[4] Hadjistavropoulos T, Hadjistavropoulos T, Psych R, Craig KD. Pain: psychological perspectives: Psychology Press; 2004.

[5] Spielberger C, Gorsuch R, Lushene R. Manuel for the state-trait anxiety inventory. Palo Alto, CA: Consulting Psychologists Press; 1970.

[6] Hollins M, Harper D, Gallagher S, Owings EW, Lim PF, Miller V, Siddiqi MQ, Maixner W. Perceived intensity and unpleasantness of cutaneous and auditory stimuli: an evaluation of the generalized hypervigilance hypothesis. Pain. 2009;141:215-21.

[7] Sullivan MJ, Thorn B, Haythornthwaite JA, Keefe F, Martin M, Bradley LA, Lefebvre JC. Theoretical perspectives on the relation between catastrophizing and pain. Clin J Pain. 2001;17:52-64.

[8] McDermid AJ, Rollman GB, McCain GA. Generalized hypervigilance in fibromyalgia: evidence of perceptual amplification. Pain. 1996;66:133-44.

[9] Ruscheweyh R, Albers C, Kreusch A, Sommer J, Marziniak M. The effect of catastrophizing self-statements on pain perception and the nociceptive flexor reflex (RIII reflex). Clin J Pain. 2013;29:725-32.

[10] Dugas M, Buhr K, R L. The role of intolerance of uncertainty in etiolody and maintenance. Generalized anxiety disorder: Advances in research and practice. New York: Guilford Press; 2004.

[11] Nelson BD, Shankman SA. Does intolerance of uncertainty predict anticipatory startle responses to uncertain threat? Int J Psychophysiol. 2011;81:107-15.

[12] Gole M, Schäfer A, Schienle A. Event-related potentials during exposure to aversion and its anticipation: the moderating effect of intolerance of uncertainty. Neurosci Lett. 2012;507:1127.

[13] Wiech K, Ploner M, Tracey I. Neurocognitive aspects of pain perception. Trends Cogn Sci. 2008;12:306-13.

[14] Carlsson K, Andersson J, Petrovic P, Petersson KM, Ohman A, Ingvar M. Predictability modulates the affective and sensory-discriminative neural processing of pain. Neuroimage. 2006;32:1804-14.

[15] Oka S, Chapman CR, Kim B, Shimizu O, Noma N, Takeichi O, Imamura Y, Oi Y. Predictability of painful stimulation modulates subjective and physiological responses. J Pain. 2010;11:239-46.

[16] Meulders A, Vansteenwegen D, Vlaeyen JW. Women, but not men, report increasingly more pain during repeated (un)predictable painful electrocutaneous stimulation: Evidence for mediation by fear of pain. Pain. 2012;153:1030-41.

[17] Lin CS, Hsieh JC, Yeh TC, Niddam DM. Predictability-mediated pain modulation in context of multiple cues: An event-related fMRI study. Neuropsychologia. 2014;64c:85-91.

[18] Dworkin RH, Turk DC, Farrar JT, Haythornthwaite JA, Jensen MP, Katz NP, Kerns RD, Stucki G, Allen RR, Bellamy N, Carr DB, Chandler J, Cowan P, Dionne R, Galer BS, Hertz S, 
Jadad AR, Kramer LD, Manning DC, Martin S, McCormick CG, McDermott MP, McGrath P, Quessy S, Rappaport BA, Robbins W, Robinson JP, Rothman M, Royal MA, Simon L, Stauffer JW, Stein W, Tollett J, Wernicke J, Witter J, IMMPACT. Core outcome measures for chronic pain clinical trials: IMMPACT recommendations. Pain. 2005;113:9-19.

[19] Freeston MH, Rhéaume J, Letarte H, Dugas MJ, Ladouceur R. Why do people worry? Personality and individual differences. 1994;17:791-802.

[20] Buhr K, Dugas MJ. The Intolerance of Uncertainty Scale: psychometric properties of the English version. Behav Res Ther. 2002;40:931-45.

[21] Bergeron J, Landry M, Bélanger D. The development and validation of a french form of the state-trait anxiety inventory.Cross-cultural anxiety. Washington (DC); Hemisphere Publishing; 1976, p 41-50.

[22] French D, Noel M, Vigneau F, French J, Cyr C, Evans R. L'échelle de dramatisation face à la douleur PCS-CF: Adaptation canadienne en langue française de l'échelle pain catastrophizing scale.L'échelle de dramatisation face à la douleur PCS-CF: Adaptation canadienne en langue française de l'échelle pain catastrophizing scale. Canadian Journal of Behavioural Science/Revue Canadienne Des Sciences du Comportement. 2005;37:181-92.

[23] Granot M, Weissman-Fogel I, Crispel Y, Pud D, Granovsky Y, Sprecher E, Yarnitsky D. Determinants of endogenous analgesia magnitude in a diffuse noxious inhibitory control (DNIC) paradigm: do conditioning stimulus painfulness, gender and personality variables matter? Pain. 2008;136:142-9.

[24] French DJ, France CR, France JL, Arnott LF. The influence of acute anxiety on assessment of nociceptive flexion reflex thresholds in healthy young adults. Pain. 2005;114:35863.

[25] Sullivan MJ, Bishop SR, Pivik J. The pain catastrophizing scale: development and validation. Psychological assessment. 1995;7:524.

[26] Roelofs J, Peters ML, McCracken L, Vlaeyen JW. The pain vigilance and awareness questionnaire (PVAQ): further psychometric evaluation in fibromyalgia and other chronic pain syndromes. Pain. 2003;101:299-306.

[27] McCracken LM. "Attention" to pain in persons with chronic pain: A behavioral approach. Behavior Therapy. 1997;28:271-84.

[28] Story GW, Vlaev I, Seymour B, Winston JS, Darzi A, Dolan RJ. Dread and the disvalue of future pain. PLoS Comput Biol. 2013;9:e1003335.

[29] Hauck M, Lorenz J, Zimmermann R, Debener S, Scharein E, Engel AK. Duration of the cue-to-pain delay increases pain intensity: a combined EEG and MEG study. Exp Brain Res. 2007; 180:205-15.

[30] Berns GS, Chappelow J, Cekic M, Zink CF, Pagnoni G, Martin-Skurski ME. Neurobiological substrates of dread. Science. 2006;312:754-8.

[31] Rhudy JL, Williams AE, McCabe KM, Rambo PL, Russell JL. Emotional modulation of spinal nociception and pain: the impact of predictable noxious stimulation. Pain. 2006;126:22133. 


\section{Table 1}

Effect of unpredictable stimulation timing on pain intensity. No interaction was found between subjective pain intensity and the predictability of the stimulation, or the order of the block. (Mean \pm Standard Error)

CSI $=$ Cue-shock interval

\begin{tabular}{|c|c|c|c|c|c|c|c|}
\hline \multicolumn{8}{|c|}{$\mathrm{CSI}=6 \mathrm{sec}$} \\
\hline \multicolumn{2}{|c|}{ Fixed block 6 sec. first } & \multicolumn{2}{|c|}{ Fixed block $15 \mathrm{sec}$ first } & \multicolumn{2}{|c|}{ Fixed block 6 sec. first } & \multicolumn{2}{|c|}{ Fixed block 15 sec. first } \\
\hline Fixed block & $\begin{array}{c}\text { Variable } \\
\text { block }\end{array}$ & Fixed block & $\begin{array}{c}\text { Variable } \\
\text { block }\end{array}$ & Fixed block & $\begin{array}{c}\text { Variable } \\
\text { block }\end{array}$ & Fixed block & $\begin{array}{c}\text { Variable } \\
\text { block }\end{array}$ \\
\hline $28.1 \pm 5.7$ & $30.0 \pm 7.5$ & $39.3 \pm 5.7$ & $38.8 \pm 7.5$ & $27.9 \pm 5.7$ & $26.8 \pm 7.5$ & $26.1 \pm 5.7$ & $31.6 \pm 7.5$ \\
\hline \multicolumn{8}{|c|}{$\mathrm{CSI}=15 \mathrm{sec}$} \\
\hline \multicolumn{4}{|c|}{$\begin{array}{l}\text { Fixed block first } \\
\end{array}$} & \multicolumn{4}{|c|}{ Variable block first } \\
\hline \multicolumn{2}{|c|}{ Fixed block 6 sec. first } & \multicolumn{2}{|c|}{ Fixed block 15 sec first } & \multicolumn{2}{|c|}{ Fixed block 6 sec. first } & \multicolumn{2}{|c|}{ Fixed block $15 \mathrm{sec}$ first } \\
\hline Fixed block & $\begin{array}{c}\text { Variable } \\
\text { block }\end{array}$ & Fixed block & $\begin{array}{c}\text { Variable } \\
\text { block }\end{array}$ & Fixed block & $\begin{array}{c}\text { Variable } \\
\text { block }\end{array}$ & Fixed block & $\begin{array}{c}\text { Variable } \\
\text { block }\end{array}$ \\
\hline $32.7 \pm 5.4$ & $30.3 \pm 7.2$ & $32.0 \pm 5.4$ & $41.9 \pm 7.2$ & $29.5 \pm 5.4$ & $28.4 \pm 7.2$ & $29.6 \pm 5.4$ & $34.5 \pm 7.2$ \\
\hline
\end{tabular}


Table 2

Effect of timing delay on subjective pain. No interaction was found between subjective pain intensity and the variation of the length of the cue-shock interval, regardless of the order of the block.

\begin{tabular}{|l|l|l|l|l|l|l|l|}
\hline \multicolumn{4}{|c|}{ Fixed block 1st } & \multicolumn{4}{|c|}{ Variable block 1st } \\
\hline $6 \mathrm{sec}$ & $9 \mathrm{sec}$ & $12 \mathrm{sec}$ & $15 \mathrm{sec}$ & $6 \mathrm{sec}$ & $9 \mathrm{sec}$ & $12 \mathrm{sec}$ & $15 \mathrm{sec}$ \\
\hline $34.4 \pm$ & $35.8 \pm$ & $37.0 \pm$ & $36.1 \pm$ & $29.2 \pm$ & $28.6 \pm$ & $27.7 \pm$ & $31.4 \pm$ \\
5.1 & 5.1 & 5.0 & 5.0 & 5.1 & 5.1 & 5.0 & 5.0 \\
\hline
\end{tabular}


Table 3

Correlation matrix. There is a strong positive link between Intolerance of uncertainty (IU) and the change in pain intensity provoked by an unpredictable timing when the cue-shock interval is long $(15 \mathrm{sec})(\mathrm{r}=0.63, \mathrm{p}=0.003)$. Also, with the pain threshold value, we can pretty much predict the value of the pain suprathreshold (intensity of the electrical stimulation to reach $30 / 100$ of pain) $(r=0.60, p=0.005)$.

\begin{tabular}{|c|c|c|c|c|c|c|c|c|c|}
\hline & IU & & & & & & & & \\
\hline Hypervigilance & 0.34 & $\begin{array}{l}\text { Hypervigilanc } \\
\text { e }\end{array}$ & & & & & & & \\
\hline $\begin{array}{l}\text { Catastrophizin } \\
\mathrm{g}\end{array}$ & 0.60 & 0.52 & $\begin{array}{l}\text { Catastrophizin } \\
\mathrm{g}\end{array}$ & & & & & & \\
\hline Trait & 0.01 & 0.29 & 0.10 & Trait & & & & & \\
\hline State & 0.64 & 0.18 & 0.43 & 0.25 & State & & & & \\
\hline $\begin{array}{l}\text { Pain variable } \\
\text { minus fixed } \\
\text { at } 6 \mathrm{sec}\end{array}$ & 0.39 & 0.40 & 0.16 & 0.60 & 0.14 & $\begin{array}{l}\text { Pain } \\
\text { variable } \\
\text { minus fixed } \\
\text { at } 6 \mathrm{sec}\end{array}$ & & & \\
\hline $\begin{array}{l}\text { Pain variable } \\
\text { minus fixed } \\
\text { at } 15 \mathrm{sec}\end{array}$ & 0.63 & 0.16 & 0.34 & 0.03 & 0.36 & 0.54 & $\begin{array}{l}\text { Pain variable } \\
\text { minus fixed } \\
\text { at } 15 \mathrm{sec}\end{array}$ & & \\
\hline Pain threshold & -0.43 & 0.17 & -0.21 & 0.03 & -0.11 & -0.34 & -0.41 & $\begin{array}{l}\text { Pain } \\
\text { threshol } \\
\text { d }\end{array}$ & \\
\hline $\begin{array}{l}\text { Pain } \\
\text { suprathreshold }\end{array}$ & -0.22 & 0.12 & -0.06 & 0.27 & 0.11 & -0.21 & -0.08 & 0.60 & $\begin{array}{l}\text { Pain } \\
\text { suprathreshol } \\
\text { d }\end{array}$ \\
\hline
\end{tabular}

Significant at $\mathrm{p}<0.05$ 


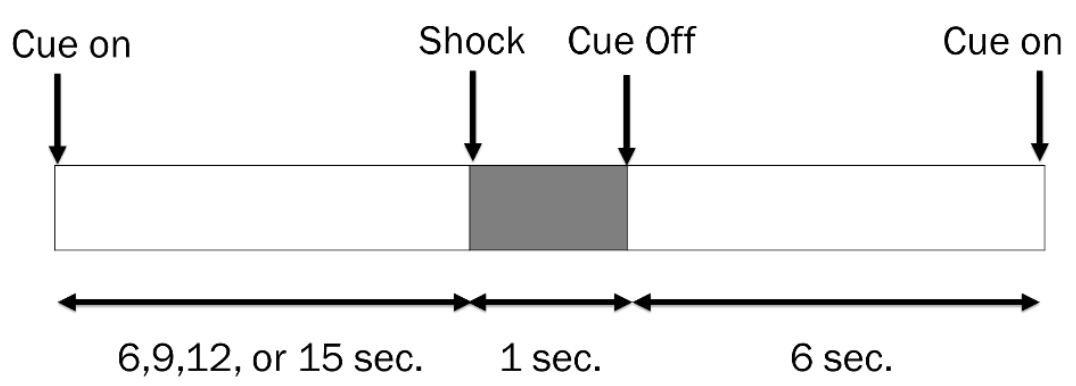

\section{Figure 1. Experimental Design}

A visual cue with a red light was presented to the participant to signal the presence of an upcoming shock (cue onset). The light remained on for the duration of the different cued shock intervals until a shock was administered. The duration varied on if the block was fixed (6 or $15 \mathrm{sec}$.) or variable (randomly sequenced $6,9,12,15 \mathrm{sec}$.). The visual cue was then turned off 1 second after the shock and remained closed for 6 seconds. During this period, participants gave their pain intensity rating. 


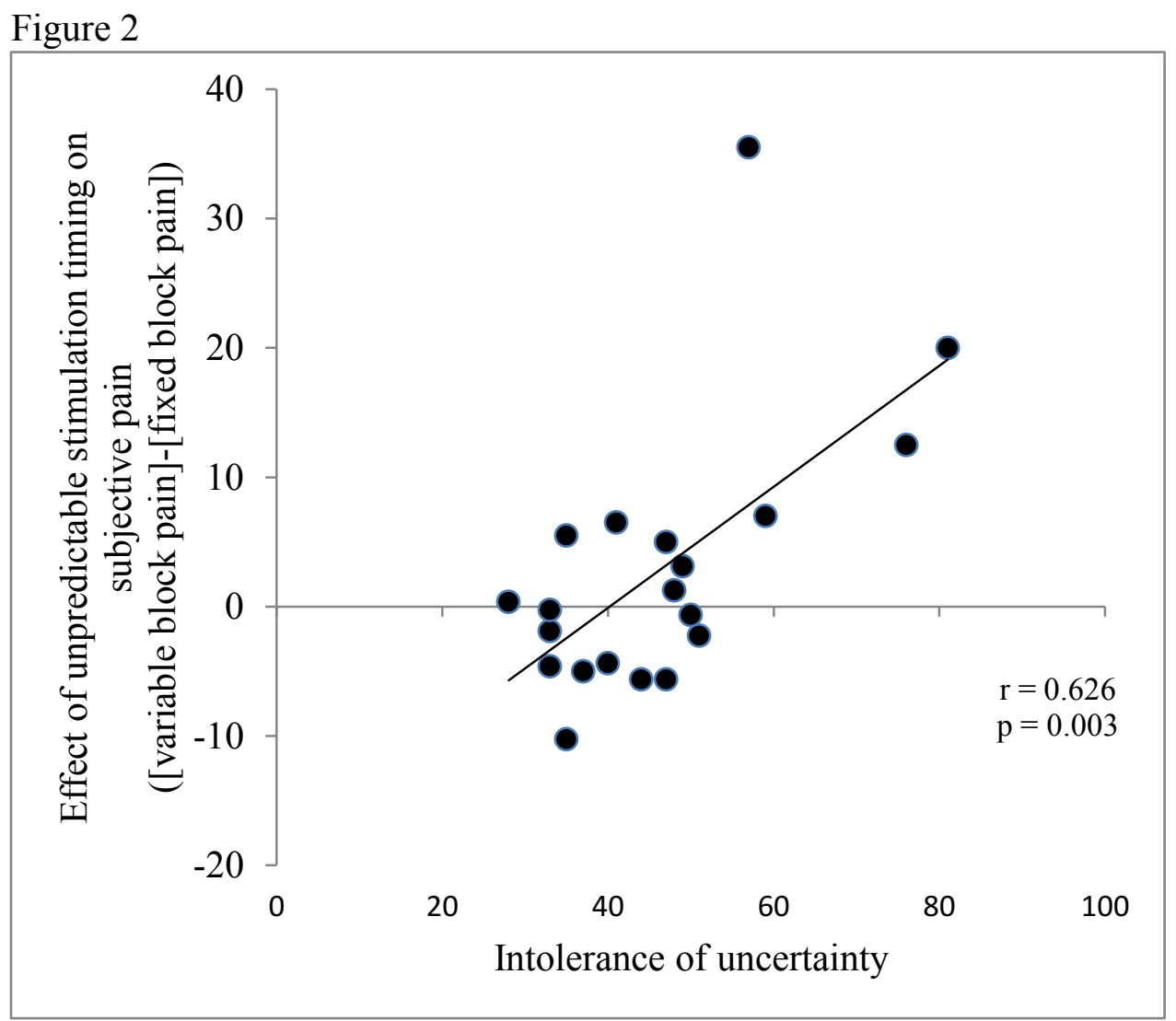

Scatter plot showing the correlation between intolerance of uncertainty and the change of pain intensity between the variable block and the fixed block for a cue-shock interval of $15 \mathrm{sec}$. A higher intolerance of uncertainty scores predicted higher pain intensity when the timing is unpredictable at $15 \mathrm{sec}$. 